\title{
La construcción de la identidad docente a partir del relato de autobiografías escolares
}

\author{
Isabel del Carmen Benítez \\ Carlos Alberto Lucero \\ (Instituto de Formación Docente Continua \\ Villa Mercedes. San Luis. Argentina)
}

\section{Resumen}

Este trabajo surge a partir de interrogantes, inquietudes, necesidades de docentes de diferentes niveles acerca de la problemática de la identidad en estos tiempos. Esto motivó la realización de una experiencia de capacitación con docentes en servicio. Los mismos fueron elaborando sus propios relatos autobiográficos que nos permitieron abordar la problemática de la identidad docente y del vínculo pedagógico.

El relato de las autobiografías escolares posibilitó una mirada hacia el interior de cada docente y al mismo tiempo analizar el recorrido durante los años de escolaridad y la motivación en la elección de esta actividad. También pudieron analizar y reflexionar sobre sus propios vínculos pedagógicos como docentes en la actualidad. De estos relatos surgieron las categorías de análisis que fueron influyendo en la elección de la carrera y su propia identidad. Estas cuestiones tienen que ver con aspectos de la relación interpersonal, el vínculo, características de personalidad, actitudes, afectos.

El relato de las experiencias de las autobiografías nos incita a pensar de otro modo, presupone un pensar diferente. Invitan a recorrer caminos sin ninguna dirección preestablecida, a construir nuestra historia, a reconocer nuestras huellas que han ido conformando nuestra propia subjetividad y nuestra identidad.

\section{Palabras clave}

Identidad Docente - Autobiografía Escolar - Narrativa - Vínculo Pedagógico - Autoconocimiento - Prácticas Pedagógicas.

\section{Summary}

This work arose from questions, concerns, needs of teachers of different levels on issues of identity in these times. This led to the completion of a training experience with teachers in service. They developed their own autobiographical stories that enabled us to address the issue of teacher identity and educational link.

The school's autobiographies allowed a look into the interior of each teacher and analyze the route during the years of teaching and motivation in the choice of this activity. 
They were also able to analyze and reflect on their own links in teaching as teachers today. These stories were the categories of analysis that were influencing the choice of careers and their own identity. These issues are related to interpersonal aspects of the relationship, personality characteristics, attitudes, feelings.

The account of the experiences of autobiographies encourages us to think differently. It invites to walk without any predetermined direction, to build our history, recognize our footprints that have shaped our identity and our subjectivity.

\section{Key words}

Teacher Identity - Autobiography School - Narrative - Educational Link - Self-Knowledge - Teaching.

\section{La construcción de la identidad docente a partir del relato de autobiografías escolares}

Este trabajo surge a partir de interrogantes, inquietudes, necesidades de docentes de diferentes niveles acerca de la problemática de la identidad de los docentes en estos tiempos. La misma atraviesa una profunda crisis, como consecuencia de políticas neoliberales que llevaron a la decadencia del sistema y a la confusión del sentido de las prácticas pedagógicas. Cabe destacar que estas políticas implementaron un programa de ajuste estructural orientado por las exigencias de los organismos internacionales, que propugnaban la descentralización de los sistemas escolares, la transferencia al sector privado, el desfinanciamiento de la educación pública, la flexibilización de la contratación docente, el vaciamiento de contenidos y la consecuente pérdida de la función primordial de la escuela, poniendo en cuestionamiento la actividad misma del docente y su propia identidad.

Esto motivó la realización de diferentes experiencias de capacitación con docentes en servicio, que permitió abordar la problemática de la identidad docente y el vínculo pedagógico.

En la construcción de la identidad profesional nos interesó conocer qué influencias tenían las historias escolares vividas. Para la reconstrucción de las mismas, se utilizó la narrativa, y esa particular forma de narrativa que se llama relato, el cual trata no sólo de hechos, ideas o teorías o hasta de sueños, temores y esperanzas, sino de hechos teorías y sueños desde la perspectiva de la vida de alguien y dentro del contexto de las emociones de alguien.

Elbaz (1990) identifica el relato como uno de los principales temas en la investigación sobre la enseñanza. El mismo constituye la materia misma de 
la enseñanza, el paisaje en el que vivimos como docentes e investigadores y dentro del cual el trabajo de los maestros tiene sentido particular.

Es interesante destacar que muchos investigadores de la enseñanza utilizan narrativas tanto en el proceso de indagación como en los informes de los resultados de la investigación. Las experiencias pasadas no se entierran como si fuesen tesoros arqueológicos, sino que el pasado es recreado a medida que se dice. A través de este diálogo narrativo entre reflexión e interpretación, la experiencia se transforma en saber pedagógico. El estudio de los relatos y las narrativas de los docentes lleva directamente al corazón mismo del saber pedagógico: la experiencia, la práctica, la reflexión y la transformación.

Respecto a la construcción de la identidad docente Viscaíno expresa "El tránsito de los sujetos por las instituciones educativas, la participación de/en las prácticas que allí tienen lugar, ofrece herramientas simbólicas y materiales para la conformación de representaciones que van configurando aspectos identitarios que definen un modo de ser y estar en ese espacio particular. Representaciones que remiten a lo que se piensa acerca de la enseñanza, del aprendizaje, del sentido que tiene la educación y la docencia como prácticas institucionalizadas..." (1).

La identidad profesional según Viscaíno, se tomará como un constructo posible de dar cuenta de las representaciones que el docente tenga de sí mismo como sujeto de una práctica específica. "Representaciones que, si bien se van configurando a lo largo de la historia del sujeto y de su tránsito por diferentes espacios sociales (diferentes instancias en las que el enseñar y el aprender han tenido lugar), se verán en este momento, resignificadas y atravesadas por lo propio de la situación actual del sujeto y del contexto" (2).

La identidad se entiende como una construcción que no está cerrada, ni se cierra en un momento particular, sino, que se encuentra abierta a la temporalidad, a la contingencia, a las diferencias que se enmarcan en el encuentro con otros signados por lo inacabado por la incompletud.

Pensar en la identidad, como lo manifiesta Emilio Porta, es situarse en la problemática del sujeto, de su accionar y su decir/se social.

La construcción de la identidad involucra la articulación de lo personal y lo social, en un movimiento, al decir de Vizcaíno, de reconstrucción mutua. "Los procesos de construcción identitaria se enmarcan en las relaciones con los semejantes, en las matrices identificatorias que se generan entre los sujetos insertos en un espacio sociocultural común. Se van configurando durante el fluir de la vida, en un movimiento signado por permanencias y novedades que no son posibles de enmarcar en un camino único y lineal, 
en un punto de llegada final. Se trata más de ser, de ir siendo. La identidad docente, en este sentido, es comprendida como parte de un proceso de subjetivación que cobra sentido en tanto proceso relacional y sociohistórico..." (3).

Algunos de los interrogantes que fueron motivo de investigación y análisis de esta problemática son: ¿Pueden los docentes intentar un cambio en las prácticas pedagógicas teniendo en cuenta la construcción de su propia identidad a partir de las experiencias narrativas de sus autobiografías escolares?, ¿Cómo influye el análisis de las autobiografías en la mejora de las prácticas pedagógicas?, ¿La instancia de la narración de las autobiografías escolares permite la reflexión sobre el vínculo pedagógico?

En la construcción del vínculo pedagógico y en el análisis de la identidad docente, se torna fundamental la cuestión del autoconocimiento. En relación a la cuestión del autoconocimiento, Jorge Larrosa plantea lo siguiente: "... El autoconocimiento, pues, aparece como algo análogo a la percepción que uno tiene de su propia imagen en tanto que puede recibir la luz que ha sido arrojada hacia atrás por un espejo.

Tendríamos así un desdoblamiento entre uno mismo y una imagen exterior de uno mismo, la que aparece en el espejo, la cual, por el efecto feliz de un cambio en la dirección de la luz, se hace visible para uno mismo como cualquier otra imagen. El autoconocimiento aparece así como una modalidad particular de la relación sujeto-objeto. Sólo que el objeto percibido, en este caso, es la propia imagen exteriorizada que, por una cierta propiedad de la luz al impactar en las superficies bruñidas, está delante del sujeto que ve..." (4).

Larrosa plantea la cuestión del autoconocimiento como un ejercicio de girar el ojo de la mente hacia adentro. Se trata de una percepción interna volviendo la mirada desde el exterior hacia el interior. El sujeto se transforma así en objeto de conocimiento, de sí mismo. Es decir se observa, se analiza, se cuestiona, se interroga. Este proceso de reconversión de la mirada hacia uno mismo es lo que desencadena nuevos y constantes ejercicios de sí y nos da la posibilidad de operar con libertad en el interior de los dispositivos escolares. Implica que uno pueda realizar cosas consigo mismo, esto es, lo que puede y debe hacer uno cuando se mira, se juzga, se analiza, se interpreta.

Bajo la forma del reflexionar y del mirarnos hacia adentro, es como tendemos a comprender la relación del docente consigo mismo y con su práctica pedagógica.

La práctica docente implica el trabajo que el maestro desarrolla cotidianamente en determinadas y concretas condiciones sociales, históricas 
e institucionales adquiriendo una significación tanto para la sociedad como para el propio maestro. Trabajo que si bien está definido en su significación social y particular por la práctica pedagógica, va mucho más allá de ella al involucrar una compleja red de actividades y relaciones que la traspasa. Respecto a la práctica pedagógica, es la que se desarrolla en el contexto del aula en el que se pone de manifiesto una determinada relación maestroconocimiento-alumno, centrada en los procesos de enseñar y aprender.

Las prácticas pedagógicas involucran un conjunto complejo de elementos; es una práctica específica que se realiza en un lugar particular, con unos estatutos, unas reglas que la rigen, en un espacio y tiempo determinado en que se exponen ciertos conocimientos, ciertos saberes, con ciertos fines sociales específicos y con vinculaciones entre diferentes sujetos que se encuentran atravesados por las relaciones de poder. En este sentido la práctica pedagógica implica o debería implicar un cierto tipo de relación del sujeto consigo mismo, lo que va determinando su constitución como sujeto de la educación a partir de sus propias experiencias vividas.

La práctica pedagógica se caracteriza por la improvisación, la espontaneidad, la inmediatez, la intuición, la percepción, etc. Dicha práctica mantiene una relación concreta con conocimientos y representaciones anteriores y marcadas históricamente, en este sentido reconstruir las biografías escolares favorecería la reflexión y hasta la transformación y mejora de la práctica.

El otro es indispensable en el vínculo pedagógico. La práctica de uno mismo entra en íntima interacción con la práctica social o, si se prefiere, con la constitución de una relación de uno para consigo mismo que se ramifica de forma muy clara con las relaciones de uno mismo a otro. Ocuparse permanentemente de uno mismo, es vivir con uno mismo, aprender, disfrutar de uno mismo, para luego ir al encuentro con aquellos que nos rodean. Esto lleva inevitablemente a repensar en la actualidad el vínculo pedagógico que aparece desgastado y resquebrajado.

El vínculo pedagógico se va conformando por las características del entorno social e institucional donde se produce dicho vínculo; por los intereses, actitudes y características de los alumnos y de los docentes y por las propias características y complejidades del conocimiento que se está enseñando. El docente y el alumno construyen el proyecto pedagógico a través de esta relación vincular. El aula es el ámbito natural donde los sujetos pedagógicos pueden realizarse o enajenarse. Cabe preguntarse: ¿Cuántos docentes se preocupan realmente porque sus alumnos aprendan a formularse preguntas?, ¿Cuántas veces lo que se enseña y no registran 
los programas escolares es lo vital, lo que perdura en la formación de los alumnos?

El docente en el vínculo pedagógico no sólo debe considerarse como portador de conocimientos sino como generador de actitudes y promotor de intereses sociales. Debe convertir la situación de aprendizaje en una situación autoconsciente a través de una sistemática crítica de los contenidos de su materia y autocrítica de los métodos con que esos conocimientos son transmitidos. En este sentido el verdadero compromiso sería considerar la acción educativa como un espacio que permita un profundo conocimiento de uno mismo y de ese modo poder formar ciudadanos críticos, democráticos, libres y transformadores de las prácticas sociales que les ha tocado vivir.

Un minucioso conocimiento de sí mismo no es otra cosa que la reconversión de la mirada del propio sujeto desde el exterior de las cosas hacia el interior, desde el mundo hacia el propio yo o lo que es lo mismo hacia la intimidad de cada uno. $Y$ es conociendo nuestro interior como se producen los grandes cambios o transformaciones que nos conducen a la reflexión permanente de nuestro obrar cotidiano. Por lo tanto, la enseñanza no debe reducirse a un simple método, ni un procedimiento de transmisión de conocimientos, ni un mero quehacer instruccional. Debe entenderse, como el espacio que posibilita el pensamiento y la reflexión acerca de sí mismo. Sólo el conocimiento que nace del interior de la persona es el verdadero conocimiento.

Este tipo de prácticas permite que los sujetos puedan constituir, definir, organizar e instrumentalizar estrategias consigo mismos y en relación a los otros en un ámbito de libertad. Estos sujetos se van transformando no en sujetos sujetados, sino en sujetos libres, capaces de generar nuevas formas de pensar y operar en su cotidianeidad.

Al respecto, Andrea Alliaud analiza la importancia formativa que representa la biografía escolar de los docentes y cómo éstas producen prácticas de libertad: "la experiencia escolar vivida en todas las etapas en las que fueron alumnos tiene un carácter formativo que tiende a imponerse sobre la formación específica" (5).

La autora plantea que los que se dedican a enseñar lo realizan en una institución por la cual han transitado. De allí la importancia de conocer el contenido de la biografía escolar ya que la historia escolar pasada está de algún modo presente organizando o configurando las prácticas y representaciones de los docentes. La biografía escolar, es decir "el periodo vivido en la escuela por los maestros siendo estos alumnos" (6), es una fase del proceso formativo que resulta enriquecedor analizarla para entender la práctica profesional y en consecuencia la identidad profesional. 
La formación es un proceso relativamente prolongado. Ferry concibe la formación como un trayecto que atraviesan los maestros y profesores durante el que transitan por diferentes experiencias e interactúan con diferentes conocimientos, enfoques y personas.

Clandinin adopta una perspectiva narrativa a partir de la cual intenta comprender las construcciones del maestro sobre la clase y la vida. El estudio de la narrativa es el estudio de la forma en que los seres humanos experimentan el mundo. "La importancia educacional de esta línea de trabajo reside en que aporta ideas teóricas sobre la naturaleza de la vida humana (en tanto que vivida) y que puedan aplicarse a la experiencia educativa (también en tanto que experiencia vivida)" (7).

En este sentido se adopta la narrativa como manera de favorecer la reflexión, la que permite al docente resignificar las ideas, asumir nuevas implicancias o considerar alternativas de transformación y mejora de la práctica. De allí la importancia de analizar las autobiografías. En ellas los docentes relataban sus experiencias escolares. Se trabaja con el relato, en el que los docentes cuentan sus historias mientras reflexionan sobre sus vivencias. "Una misma persona está ocupada, al mismo tiempo, en vivir, en explicar, en re-explicar y en re-vivir historias" (8).

Al contar historias sobre la escuela, sus prácticas pedagógicas, sobre los aprendizajes de los alumnos, sobre las vicisitudes e incertidumbres escolares, sobre las estrategias de enseñanza que adoptan, sobre sus reflexiones, los docentes hablan de sí mismos, de sus sueños y de sus realizaciones. $Y$ en ese narrar-se los maestros reconstruyen su identidad docente. Narrando sus prácticas escolares los maestros cuentan sus propias biografías profesionales, sus perspectivas e impresiones acerca de lo que consideran una buena práctica de enseñanza, el papel de la escuela en la sociedad contemporánea, sus propios lugares en la enseñanza y en la escuela, lo criterios de intervención curricular, los supuestos que subyacen a las formas con que evalúan los desempeños de los estudiantes y los suyos propios. Con sus historias muestran parte del saber pedagógico práctico y muchas veces oculto, que construyen y reconstruyen sus prácticas profesionales.

La experiencia narrativa brinda la posibilidad de volver reflexivamente sobre lo hecho, usando la escritura como una vía para la crítica y transformación de la propia práctica. Estos relatos de los docentes forman parte de las dimensiones subjetivas y permiten la reflexión, la interpretación y el intercambio con los demás sujetos pedagógicos. En este sentido el docente es un constructor activo de su propio texto y de los saberes que lo han configurado. El relato de experiencias tiene la huella del que narra. Al 
decir de Jorge Larrosa: "Al narrar-se, uno dice lo que conserva de lo que ha visto de sí mismo. Por otra parte, el decir-se narrativo no implica una descripción topológica, sino una ordenación temporal. Así, el narrador puede ofrecer su propia continuidad temporal, su propia identidad y permanencia en el tiempo en la misma operación en la que construye la temporalidad de su historia" (9).

Los procesos que implican el narrar-se, pretenden contribuir a una redefinición de los modos de conocer, problematizar y recrear sus saberes, permite pensar sobre sus propias huellas que han dejado una impronta en sus prácticas escolares. A través de esas narraciones los docentes autores descubren sentidos pedagógicos, que antes estaban ocultos, elaboran sus propios discursos, lo comunican, lo critican, lo comparten y lo vuelven a construir. El narrador toma de la experiencia, la suya propia o la transmitida. Y la torna a su vez, en experiencia de aquellos que escuchan su historia. La marca del sujeto es lo que distingue la narración de la información y lo que otorga al relato un carácter potencialmente provocador o convocante que puede mover, inspirar, solucionar si logra seducir a otros. El sujeto de la experiencia es un sujeto pasional, receptivo, abierto, expuesto.

Las narraciones más que imponer, suscitan cursos de acción y respuestas diversas. Proponen, provocan, intrigan, sorprenden y dejan pensando al lector. Son, de este modo, potencialmente ricas para producir prácticas, pensamientos y reflexiones en tanto convocan, interpelan o provocan. Intentan recuperar el saber de la experiencia. Ese saber tiene que ver con lo que somos, con nuestra formación y nuestra transformación y de los demás sujetos. "Es contando historias, nuestras propias historias, lo que nos pasa y el sentido que le damos a lo que nos pasa, que nos damos a nosotros mismos una identidad en el tiempo" (10).

Este proceso de narrar-se desencadena un pensar que es autónomo e independiente. Es libre, en un sentido amplio, es capaz de reflexionar sobre su propia experiencia y sobre su situación en el mundo. Está preparado para evaluar sus prácticas y en consecuencia reconocer su propia identidad. Este impulso a pensar por nosotros mismos surge en proporción a nuestra disposición a discutir, deliberar con los otros, construyendo ideas, aportando y analizando situaciones problemáticas, ayudándose unos a otros a formular preguntas y a ampliar los puntos de vista. "La construcción y la transformación de la conciencia de sí dependerá, entonces, de la participación en redes de comunicación donde se producen, se interpretan y se median historias" (11). 


\section{Experiencias de docentes en servicio}

Para la conformación de esta experiencia de trabajo, que permitió abordar la identidad de los docentes a partir de revisar su autobiografía escolar, se recuperaron los relatos de los mismos.

Las autobiografías permiten ver de qué manera y quiénes conformaron la historia escolar y cómo ésta influye en la construcción de la identidad docente. En este sentido se puede vivenciar el espacio de la práctica pedagógica como un espacio que se pueda transformar y mejorar.

En las autobiografías analizadas se tuvieron en cuenta dos categorías el escenario, es decir el lugar donde la acción ocurre, donde se viven las historias; y el tiempo, se refiere a la vida en movimiento, es la historia con un comienzo, mitad y final. El análisis de la biografía escolar no es solamente el recuerdo de un maestro o profesor, es todo lo que los docentes aprendieron en la institución escolar.

La biografía escolar, es decir, el período vivido en la escuela por los maestros siendo alumnos, es una fase del proceso formativo que resulta enriquecedor analizarla para entender la práctica profesional y en consecuencia la identidad profesional. Si bien cada biografía se analiza a partir de lo que "dice" cada docente, no se desconoce que las mismas son producto de una construcción social en la que se conforman.

Alliaud se refiere a la misma como "aquello que se vive y experimenta en determinadas circunstancias en las que se producen los aprendizajes de contenidos formalizados. Es lo aprendido informal o implícitamente en la prolongada estadía que uno pasa por las instituciones escolares" (12). Lortie lo denomina "aprendizaje por observación", Jackson "aprendizaje adicional", cualquiera de estas nominaciones, estos aprendizajes dejan una huella que ejercen influencias al momento de enseñar. En este sentido y según lo planteado por Alliaud, la biografía escolar pasa a ser la primera fase de la formación docente.

La experiencia formativa remite a todo lo que se aprendió, en tanto se vivió. Es lo aprendido en tanto nos pasa como sujetos. Forma parte de la trama de nuestras vidas.

Los relatos autobiográficos que presentamos a continuación corresponden a docentes en servicio de Nivel Inicial, Primario, Secundario, y Superior de la Ciudad de Villa Mercedes (San Luis), en el período 2006 y 2008. El análisis realizado de las autobiografías permite visualizar algunas cuestiones que aparecen reiterándose durante el recorrido de la escuela vivida.

Del análisis de las autobiografías surgen las siguientes categorías: aspectos vinculados a lo personal: el vínculo, características de personalidad, actitudes, valores, afectos y, aspectos de la formación profesional: 
maneras de enseñar de los docentes y maneras de enfrentar las situaciones didácticas.

En relación al primer aspecto que se vincula con lo personal, aparece lo siguiente: respecto de las características de personalidad, recuerdan aquellos docentes abiertos o con una actitud de apertura, acogida y cuidado que se destacaban por sus afectos, en términos de los docentes, eran "dulces", "cariños", "cálidos", Ilenos de "ternura" que los "acompañaban" permanentemente. Por otro lado, estaban los docentes que dejaron sus huellas por ser "cerrados", que se dedicaban a "gritar", y ser "injustos". En este sentido, en el relato de las autobiografías, los docentes hacen referencia a actitudes discriminatorias, por ejemplo, cuando dividían a los alumnos en categorías (muy buenos, buenos, regulares, malos) y los ubicaban en determinados lugares dentro del espacio áulico. Actitudes que se vinculaban con la ridiculización y la burla, y hasta docentes que ignoraban totalmente a sus alumnos.

Otro de los recuerdos negativos, se vincula con la comparación con los compañeros de grado que podían desarrollar mejor algunas habilidades, como una docente lo expreso: "los otros siempre son mejores que yo".

Varios docentes en el relato de sus experiencias escolares se refirieron a los valores o principios morales que encarnaban sus maestros y profesores, y que movilizaron en ellos la admiración e incluso el deseo de imitación. Destacaron entre otros la rectitud, el compromiso con la tarea, la responsabilidad, disciplina, la exigencia, esfuerzo, todo esto necesario para ser alguien en la vida y poder desarrollar un proyecto personal. En general, recuerdan estos aspectos como muy importantes y que conforman la manera de ser docente, la manera de enseñar de ellos actualmente. Se destaca especialmente la exigencia como aspecto fundamental de los docentes. Se vincula a la exigencia consigo mismo y con los alumnos.

En general destacan la rectitud, la exigencia, la paciencia y el amor como características fundamentales del oficio de enseñar. Hay algunos relatos que recuperan aspectos vinculados con la vestimenta, presencia, perfumes y elegancia de los docentes.

En relación al segundo aspecto, éste se vincula con maneras de enseñar y de enfrentar las situaciones didácticas. La dimensión pedagógica queda relegada ante los aspectos antes mencionados, pero varios relatos hacen referencia a la manera de explicar que tenían sus docentes y que se vinculaba con buscar formas para que los alumnos comprendieran temáticas. En siete autobiografías se destaca que los profesores que tenían autoridad intelectual y epistémica, porque sabían la materia, la explicaban bien y hacían que los alumnos terminaran "gustando" de disciplinas como 
la física, la matemática, entre otras. Y en algunos casos los docentes que relataron esto, terminaron siendo profesores de esas disciplinas.

También hay dos relatos que hacen referencia a docentes que tenían problemas físicos. El primero en la Universidad, con cáncer de garganta que para dar clase usaba megáfono, era excelente profesor, enseñaba con pasión al decir del docente que relató la experiencia. Este docente ejerció tal influencia que actualmente enseña la misma disciplina que daba ese profesor. El segundo caso es de un profesor ciego que reconocía a sus alumnos por la voz, la huella que dejó en sus alumnos fue el deseo permanente de superación.

En definitiva y compartiendo con Alliaud "no sólo están formados por la preparación profesional, sino que fueron formados o formateados y por qué no escolarizados por la experiencia vivida como alumnos. Lo que se aprende como alumno, afirma Lortie, se generaliza y convierte en tradición, constituyendo así una poderosa influencia que trasciende las generaciones" (13).

Las autobiografías permiten ver de qué manera y quiénes conformaron nuestra historia escolar y cómo ésta influye en la identidad docente favoreciendo la reflexión sobre el vínculo pedagógico. En este sentido, se puede vivenciar el espacio de la práctica pedagógica como un espacio modelable.

En síntesis, del análisis de las autobiografías se puede evidenciar que el recuerdo positivo que los docentes tienen de sus maestros y profesores, son de aquellos que los hacían sentir personas, los valoraban como eran, y les enseñaban con exigencia, compromiso y pasión. En definitiva, aquellos que les enseñaron bien, que les hicieron aprender comprensivamente y que les dieron posibilidades de pensar en un proyecto de vida (como era el de continuar con la misma profesión de esos docentes).

En tanto que, el recuerdo negativo como fueron las situaciones de vergüenza, mal trato, castigo, humillación y comparación conforman las vivencias sufridas que, si no han sido objeto de un análisis formativo ni de reflexión consciente, pueden incidir negativamente en los que actualmente se dedican a la docencia.

\section{Reflexiones finales que permitan seguir pensando}

El proceso del relato de las autobiografías escolares implica un conocimiento profundo de uno mismo, del lugar y del momento en que un sujeto desarrolla su práctica y, al mismo tiempo, la libertad de actuar sobre el otro produciendo cambios en su interior. El recorrido por las instancias planteadas anteriormente, permitió a los docentes redescribir y resignificar 
su identidad profesional en este contexto posmoderno y producir nuevas formas de subjetivación. Es en contacto con este tipo de prácticas de enseñanza donde se construye y modifica la experiencia que los individuos tienen de sí mismos; es el espacio donde se transforman los seres humanos en sujetos sujetados o en sujetos libres de expresión, libres de pensamientos, libres de actuar.

La intervención del docente en la práctica pedagógica es clave, ya que es él quien tiene a su cargo la función de conducir aprendices, generar ideas, organizar actividades de exploración o de descubrimiento poco o nada estructuradas. Esto quiere decir, que el docente debe tratar que sus alumnos sean activos y participativos en sus propios procesos de subjetivación, buenos observadores de sí mismos y de su hacer y ser en relación con los otros. En este sentido, en el análisis de las autobiografías de las experiencias realizadas, el recuerdo positivo es de aquellos docentes que los valoraban como personas, y les enseñaban con compromiso y pasión, en definitiva, los que les ayudaron a construir el proyecto de vida. En tanto que los recuerdos negativos se relacionan con vivencias de mal trato y humillación.

Es fundamental que el docente aprenda a cuestionar sus conocimientos, aprenda a pensar por sí mismo. En este sentido se puede hablar de un docente con sentido crítico en relación a sí mismo, en relación a los conocimientos que imparte y en relación al contexto social donde se produce no sólo el conocimiento, sino también su propia práctica. Este sentido crítico ayuda a despertar la creatividad y la autonomía que necesitan los alumnos para desenvolverse. Como señala Jorge Larrosa: “... la crítica es un trabajo fundamentalmente moral, un trabajo que implica, además, una cierta confianza en la posibilidad de transformar 'eso' que, según parece, transmiten las formas dominantes de educación... Pero, ¿qué es la crítica sino el cuestionamiento permanente de las formas de experiencia que nos constituyen?... La crítica no es un pretexto, momento mediador. Es, por el contrario, un ejercicio permanente de contestar las evidencias y de vivir y pensar en las inseguridades del presente" (14).

Este cuestionamiento no solamente le compete al alumno, sino también al maestro, ya que ambos sujetos son los que conforman y dan sentido al vínculo pedagógico. Pero este cuestionamiento no debe centrase únicamente en relación a los saberes, discursos, conocimientos; sino que debe extenderse a las maneras de actuar, a los gestos, comportamientos, a las actitudes, a los desenvolvimientos, en fin, a todo aquello que va conformando la ontología misma de cada sujeto. Estos son algunos de los 
aspectos que en las autobiografías analizadas configuran los recuerdos más significativos y relevantes.

Para que esto pueda lograrse es necesario que el maestro experimente un conjunto de prácticas que transformen su experiencia de sí. Y esto se logra cuando el docente puede incursionar en su propia historia personal, sus afectos, sentimientos, significaciones, valores, ilusiones y proyectos. $Y$ es aquí donde empieza a construirse este proceso complejo como el narrar-se que surge cuando el docente puede distanciarse, objetivarse de lo que relata para ver la experiencia en su conjunto y verse a sí mismo como constructor de su propia práctica pedagógica.

Para finalizar, es interesante reflexionar sobre dos cuestiones que remiten a la experiencia según Skliar en el texto: La educación [que es] del otro: “... Y en tanto experiencia, en tanto sentido de experiencia, ella puede remitirnos a dos cuestiones que nos parecen aquí interesantes: una, la de experiencia no sólo como reflejo y/o expresión de lo vivido, sino también como algo que está ciertamente emparentado con lo pensado; la otra, la de experiencia ya no como información y/o opinión, sino en un sentido de implicancia personal, subjetiva y pasional (esto es: aquello que me pasa; aquello que nos pasa)" (15).

El relato de las experiencias de las autobiografías nos incita a pensar de otro modo, presupone un pensar diferente. Invitan a recorrer caminos sin ninguna dirección preestablecida, invitan a construir nuestra historia, a reconocer nuestras huellas que han ido conformando nuestra propia subjetividad y nuestra identidad. Y es en este punto, cuando establecemos un encuentro con el otro que nos invita a construir juntos nuevas experiencias pedagógicas.

Hacer de este proceso de narrar-se una práctica constante en nuestro transitar como docentes, es poder desenvolvernos en el interior de espacios o ámbitos de libertad y al mismo tiempo convertirnos en sujetos libres para pensar, libres para actuar, que no es otra cosa que el objetivo fundamental que debe orientar a los maestros en sus prácticas pedagógicas actuales. Esto implica estimular la capacidad de razonar y desarrollar una conciencia crítica. Aquí los sujetos pedagógicos tienen la posibilidad de ver y pensar de otro modo, de cuestionarse cada una de sus actividades. Implica tomar otra postura frente al conocimiento; no en relación a una verdad que le es impuesta desde afuera, sino en relación a una verdad sobre sí mismo que el mismo debe contribuir activamente a producir. Aquí los procesos de enseñanza y de aprendizaje se desarrollan dentro de ámbitos de libertad donde se pueda ejercitar el pensamiento, transformar el entorno y poder transformarse a sí mismo. 
Este tipo de prácticas orientan a los sujetos a la elaboración de una determinada forma de problematización de las mismas en las que se encuentran inmersos con la finalidad de construir y transformar la experiencia de sí. Como señala Jorge Larrosa: "Algunas prácticas pedagógicas, entonces, incluyen técnicas encaminadas a establecer algún tipo de relación del sujeto consigo mismo, a hacer determinadas cosas con esa relación $y$, eventualmente, a transformarla... se trata de producir y mediar ciertas 'formas de subjetivación' en la que se establecería y se modificaría la 'experiencia'que uno tiene de sí mismo" (16). El vínculo pedagógico no puede ser visto ya como un espacio neutro o aproblemático, sino por el contrario como un espacio de posibilidades para el desenvolvimiento del sujeto y la mejora del mismo. Un espacio donde se puedan generar constantemente nuevas formas de experiencia de sí, como una manera de tener en cuenta el modo particular en que se posiciona cada sujeto.

Crear este nuevo ámbito en las diferentes prácticas pedagógicas, nos facilita ver las cosas de otro modo. Y precisamente en la cotidianeidad educativa es donde se ponen en juego diferentes formas de subjetivación. De cada uno de nosotros depende el poder realizar y hacer que los demás realicen una mirada distinta de la que veníamos haciendo. Pero no sólo realizar una mirada distinta, sino de poder lograr cambiar la disposición de aquellas cosas que están determinando nuestro modo de actuar.

Esta nueva manera de pensar el vínculo pedagógico permite recuperar lo heterogéneo, lo particular, las discontinuidades, las formas concretas en que se construyen los significados sociales en las prácticas educativas. Genera una relación de reciprocidad ya que al ocuparse de sus prácticas como docente y repensarlas cotidianamente, comparte con sus alumnos la misma experiencia despertando en ellos el ejercicio del cuestionamiento, que luego redunda en el docente como un elemento gratificante que es propio de las prácticas de libertad. Por lo tanto si un docente se ocupa de sí mismo como es debido, es decir, si sabe ontológicamente quién es, si es consciente de lo que es capaz, si conoce sus limitaciones, si puede dar cuenta del origen de sus saberes, de su paso por las diferentes instituciones, de la constitución de su propia subjetividad, podrá entonces establecer un encuentro con el otro, en un contexto de libertad y reciprocidad.

\section{Notas Bibliográficas}

(1) Viscaíno, A. M. La construcción de la identidad docente: sujetos, instituciones y prácticas sociales en Chapato M. y Errobidart A. (2008). Hacerse docente. Buenos Aires, Miño y Dávila, p. 78. 
(2) Vizcaíno A. M. (2008) Ibid, p. 79.

(3) Vizcaíno A. M. (2008) Ibid, p. 79.

(4) Larrosa, J. (1995) "Escuela, Poder y Subjetivación". "Tecnologías del Yo y Educación". Ediciones de la Piqueta, pp. 294-295.

(5) Alliaud, A. (1999) "La biografía escolar de los maestros. Una propuesta de abordaje. Reseñas de investigación”. Propuesta educativa 23, p. 89.

(6) Alliaud, A. (1999 b) "La experiencia escolar de maestros inexpertos. Biografías. Trayectorias y práctica profesional". Revista Iberoamericana de Educación, p. 35.

(7) Ibid, p. 18.

(8) Ibid, p. 22.

(9) Larrosa, J. (1995) Op cit., pp. 308-309.

(10) Larrosa, J. (1995) lbid, pp. 308-309.

(11) Larrosa, J. (1995) lbid, pp. 308-309.

(12) Alliaud, A. Op. cit., p. 19.

(13) Alliaud, A. Op. cit., p. 8.

(14) Larrosa, J. (1995) Op cit. p. 13 y 15.

(15) Skliar, C. (2007) "La educación [que es] del otro". Argumentos y desierto de argumentos pedagógicos. Capítulo 2 De la herencia y los herederos. Buenos Aires-México, Editorial noveduc, pp. 30-31.

(16) Larrosa, J. (1995) Op. cit., p. 282.

\section{Referencias bibliográfícas}

- Alliaud, A. (1998) "El maestro que aprende", en Ensayos y experiencias. Ediciones Novedades Educativas.

- Alliaud, A. (1993) "Los maestros y su historia: los orígenes del magisterio argentino". Buenos Aires, Centro Editor de América Latina.

- $\quad$ Alliaud, A. y Duschatzky (comp). (1992) Maestros. Formación, práctica y transformación escolar. Buenos Aires, Miño y Dávila editores.

- Chapato, M. Errobidart, A. (comp.) (2008) "Hacerse docente”. España, Editorial Miño y Dávila.

- Foucault, M. (1987) "Hermenéutica del sujeto". Madrid, Ediciones la Piqueta.

- Foucault, M. (1995) "El Yo Minimalista y otras conversaciones". Biblioteca de la mirada.

- Larrosa, J. (1995) "Escuela Poder y Subjetivación". Madrid, Ediciones la Piqueta.

- Skliar, C. (2007) "La educación [que es] del otro". Argumentos y desierto de argumentos pedagógicos. Buenos Aires-México, Editorial noveduc.

- Viscaíno, A. (2008) El aprendizaje de la práctica docente. Ingreso a la docencia: entre mimetizarse, parecerse, diferenciarse. Facultad de Ciencias Sociales. Universidad Nacional del Centro de la Provincia de Buenos Aires. 